\title{
La peur du vide
}

\section{Michel Matter}

Dr méd., vice-président de la FMH, département Prestations et développement professionnel

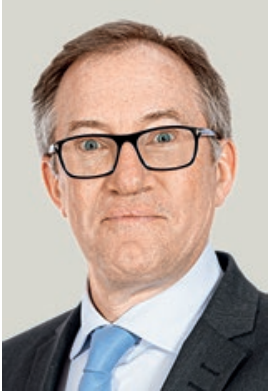

La crise sanitaire, sociale et économique que nous traversons tous ensemble est longue et pesante. Avec l'espoir que la vaccination des plus vulnérables et fragiles de la société apporte enfin une lumière au bout du tunnel. Les dernières semaines et les interventions fédérales montrent que le printemps et possiblement l'été seront difficiles. Chacune et chacun est impacté dans son quotidien tant sur les plans professionnels que privés.

Les étudiants, quelles que soient leurs facultés, poussent un cri du cœur qui doit être entendu. Quasiment plus de rapport avec les autres étudiantes et étudiants, pas d'interaction directe avec leurs professeurs et pour les études de médecine moins de pratique clinique. La pandémie a tout chamboulé et, avec elle, le risque d'une formation plus limitée et de moins bonne qualité existe. Rien ne remplace l'émulation estudiantine et la pratique directe qui permet une acquisition des compétences cliniques et reste le véritable cœur de la formation médicale. Passer de la théorie à la pratique, du document en ligne à la réalité de la palpation et de l'auscultation, est le grand moment des études de médecine, celui où enfin la blouse blanche et le métier deviennent réalité.

\section{Rien ne remplace le passage de la théorie à la pratique, du document en ligne à la réalité de la palpation et de l'auscultation.}

Pour les étudiants aînés, ils se retrouvent souvent dans des services où le rythme s'est accéléré, où le stress est palpable et où l'épuisement menace. C'est une immersion rapide et certains se retrouvent au cœur d'un environnement nouveau et sans véritables repères. Les responsabilités sont vite acquises, l'intégration dans les unités doit se faire au pas de charge car les internes et les chefs de cliniques sont moins disponibles et fatigués. La notion de responsabilité vis-à-vis de la société et de la communauté est relevée et leur engagement dans des conditions difficiles est une aide précieuse dans la gestion de l'ensemble des patients hospitalisés en raison du coronavirus ou non.

L'engagement des étudiants dans ces conditions difficiles est une aide précieuse dans la gestion de l'ensemble des patients hospitalisés.

L'impact psychique de la crise est immense. Cette crise sanitaire fait des ravages dans toutes les couches de la société. Elle frappe de plein fouet les étudiants qui sont en plein désarroi. La jeunesse se sent oubliée, mise à l'écart. Son cri de détresse est inquiétant. C'est l'absence de perspective claire sur l'avenir proche, mais aussi l'isolement social, la peur de faire des études au rabais et la crainte d'être questionné lors d'examens sur des matières peu enseignées ou assimilées qui rendent les étudiants angoissés et stressés. Nous nous devons d'entendre leurs souffrances rapportées par les médias, mais également de façon de plus en plus marquée par les psychiatres et les médecins de premier recours.

Comme thérapeute, nous sommes confrontés tous les jours à des patients qui disent leur déprime, leur solitude, leur mal-être ou encore leur ras-le-bol d'une situation perçue sans fin. Les addictologues dénoncent une explosion de la consommation de substances psychoactives et illégales. Les pédopsychiatres, les psychiatres et les médecins de premier recours tirent également la sonnette d'alarme. La crise sanitaire a entraîné une crise économique et sociale, mais aussi psychologique. Notre rôle est de protéger, d'entourer mais aussi de dénoncer une situation dramatique où la jeunesse se sent sacrifiée. 